\title{
Bemerkenswerte übereinstimmende kolloide Metallreaktionen, Spektralanalyse und Blutfarbstoff.
}

\author{
Von Eduard Richter (Hamburg). \\ (Eingegangen ath 18. Jub 1919.)
}

Unter der groben Anzahl von tierischen; pflanzlichen und rein chemischen Körpern, welche ich innerhalb zwölf Jahren (mit Kriegsunterbrechung) untersuchte, ist eine Reihe besonders interessierender Stoffe, welche bisher unbekannte kolloide Reaktionen hervorbrachten. Ueber einige dieser Kötper und ihre Reaktionen habe ich bereits in der Deutsch. med. Wochenschr. Nr. 26, in der Med. Klinik Nr. 28 und einer folgenden $\mathrm{Nr}$. (1919) berichten können. In diesen Arbeiten ist z. B. das Adrenalin bzw. Suprarenin in einer Lösung von 1:1000, ja in I_ösungen von 1 bis zu 80 Millionen noch als wirksam geschildert, und Kolloidreaktionen angegeben, welche sich in den tierischen Körperorganen, Blut und Harn, als neue Reaktionen finden lassen.

Wenn ich hiet andere neue reduktive Körper zur Erwähnung bringe, so berühre ich nur kurz jene von mir gefundenen Stoffe, der Hypophyse und der Schilddrüse, welche ich entsprechend zum Adrenalin, Hypophysalin und Thyrealin nannte.

Die anderen von mir untersuchten Körper führe ich nur insoweit an, als sie einigermaben gute Kolloidreaktionen geben und werde zum Schlub erst die hervorragendsten Stoffe von grö̈ter kolloidchemischer Wirkung nennen. $\mathrm{Zu}$ solchen von mir untersuchten Körpern gehören : Agar, Akrolein, Adrenalin, Alizarin, Alloxan, Alkaloide, Amidoazobenzol, Amidophenolchlorhydrat, Aminobenzoesäure, Anilin, Anthrazen, Antifebrin, Asparaginsäure, Aspirin, Atoxyl, Atropin, Benzidin, Benzophenon, Brenzkatechin, Cholesterin, Diazetyldioxim, Dimethylamidobenzaldehyd, Dimethylparaphenylendiaminchlorhydrat, Diphenylamin, Guajakol, Guanin, Glyzerin, Glykocholsaures Natrium, Glykol, Harnsïure und ihre Salze, Hexamethylentetramin (ohne Reaktion), Hippursäure, Hydrochinon, Hypoxanthin (ohne Reaktion), Kaffee, Koffein, Kokain, Kognak, Kreatin (ohne Reaktion), Kreatinin, (ohne Reakion), Kymol, Lezithin. Morphin, Naphthol, Naphthylamin, Natriumzitrat, Natrium nucleinicum, Neosalvarsan, Novokain, Oleinsaures Natrium, Phenazetin,Phenylendiamin, Piperazin, Phlorizin, Pyridin, Pyrodin, Resorzin, Rhodansalze, Salvarsan, Sulfanilin, Suprarenin, Strychnin, Taurocholsaures Natrium, Tee, Theo- bromin, Thioharnstoff, Thymol, Toluol, Toluidin, Toluylendiamin $(1: 3: 4)$, Tyrosin, Wein, Weinsäuresalze, die Zucherarten (besonders. Milchzucker), Zyankalium.

In der vorstehenden Reihe von Körpern habe ich solche ohne Reaktion nur deshalb angeführt, weil es mir in Sinne der Untersuchungen beachtlich erschien. Körper, welche im Wasser allein nicht reagierten, habe ich auberdem in Alkohol, Azeton, Ligroin, ferner in schwach alkalischen und sauren Lösungen einer Prüfung unterzogen. So gibt es Körper, wie z. B. das Kreosot, Guajakol, Cholesterin usw., welche in schwach alkalischer Lösung z. B. durch Zusatz von . Kalkwasser oder dünner Kaliumkarbonatoder Natriumkarbonatlösung gut reagieren. $E_{s}$ würde mich heut $\mathrm{zu}$ weit führen, alle die Kolloidreaktionen dieser angeführten Körper genauer zu schildern; ich möchte mich zunächst nur zumeist an die Stoffe halten, welche die bemerkenswertesten Reaktionen geben. Auch habe ich davon abgesehen, bisher bekannte Stoffe zu zitieren, wie z. B. Hydroxylamin, Formaldehyd, Ameisensäure, Hydrazinhydrat, unterphosphorige Säure, phosphorige Säure.

Die Körper nun von besonders hervorragender Wirkung sind außer dem Suprarenin, Lezithin, Alloxan, gallensaure Salze, Tyrosin, Atoxyl, Salvarsan, Neosalvarsan, Tannin, Pyrodin, Glyzerin, Toluidin, Toluylendiamin, und vor allem das Dimethylparaphenylendiaminchlorhydrat.

Die Darsteilung dieser Kolloidreaktionen ist nach meiner langjährigen Erfahrung am besten so zu gestalten, daf man zu $10 \mathrm{ccm}$ dest. Wassers etwas von dem zu beobachtenden Stoff hinzufügt, darin löst (oder in Alkohol. Azeton usw. löst) und dann zur Feststellung der Reduktionskraft des betr. Stoffes einige $(3-5-10)$ Tropfen einer 1 proz. Goldchloridlösung (Aurum flavum, nicht fuscum) hinzufügt oder einige Tropfen einer 1,1 proz. Goldnatriunchloridlösung, ev. mit vorherigem alkalischen Zusatz. Sieht man bei dieser streng innegehaltenen Reilienfolge rote oder amethystfarbene oder blaue oder braune Metallreduktion eintreten, so eignet sich der Körper zur weiteren Untersuchung. Heibe Lö. sungen reagieren ungefähr dreimal so kräftig wie kalte, was bei dem Zusatz von Metallösung quantitativ zu berücksichtigen ist. Die Reduk- 
tionskraft der einzelnen Körper ist nicht nur qualitativ verschieden, sondern auch quantitativ und zeithich, so dab man gut tut, eine des weiteren $z u$ beobachtende Reaktion noch bis $z u$ acht Tagen, ja bis zu 14 Tagen ruhig stehen zu lassen. Mit Hilfe der genannten Körper gelingt es, kirschrote; himbeerfarbene, violette, seegrïne, blaue, braune Metallkolloidreaktionen je nach der Reduktionskraft des Stoffes hervorzubringen, wobei das betr. Metall (z. B. Gold, Kupfer) in roten, bläulichen oder schwarzen Partikelchen durch spontanes Abstehen oder längeres Zentrifugieren zu Boden fallen kann.

Auch hier kommt es mir heute nicht darauf an, die qualitativen und quantitativen Verhältnisse genaucr zu schildern. Der bisher bestehenden Meinung, daB Kolloidreaktionen eine Hysteresis zeigen, bald kommen können, bald nicht, möchte ich den Satz gegenüberstellen, dab sie ebenso wie jede andere chemische Reaktion einer stets normativen Gesetzmäbigkeit folgen. Es kommt dabei nur auf die richtige Dosierung der einzelnen Reaktionskörper an. Die Kolloidreaktion ist eben eine Reaktion geringerer Quantitäten, wie wir sie dem gegenüber an den allgemeinen chemischen Reaktionen kennen; daraus resultiert die Forderung genatuester Dosierung eigentlich von selbst.

Einzelne chemische Körper bringen, wie z. B. das Toluylendiamin, Salvarsan in Goldlösungen, Silberlösungen usw. braune Reduktionen hervor, sie sind aber imstande, z. B. in einer violetten flloxanreaktion nachträglich hinzugesetzt, himmelblaue Goldlösungen hervorzubringen. Warum die Molekularkomplexe der Metalle von einzelnen chemischen Körpern feiner oder gröber dispers hervorgebracht werden, ist in ibrer chemischen Konstitution begründet. Hier werden uns genauere Beobachtungen noch weitere befriedigende Resultate über Metallmolekularkomplexe und Molekulargewicht hrmgen können.

Im Allgemeinen will ich gleich vorausschicken, dab die roten dispersen Metallphasen spektralanalytisch Absorptionsstreifen in gelb und grün geben, whihrend die braunen $(z . B$. des Goldes, Sibers, Palladiums, Platins, Kupfers usw.) den visletten Teil des Spektrums verdecken: Auch dieses Verhaiten gegenüber dem Spektrum wird uns manche Aufschlüsse über die Molekularkomplexe der Metalle im allgemeinen geben.

Auch dariber werden wir uns orientieren können, ob die Reduktionswirkung bis zum metallischen Niederschlag immer über die Ent- stehung von Komplexsalzen führt oder nich: (Ehrlich \& Karrer, Arsenornetallverbindungen). Ich gebe vier Reaktionskörper in ihren kolloidchemischen Reaktionen hier genauer an, nämlich 1. das Adrenalin, 2. das Alloxan. 3. das Tamin und 4. das Dimethylparapheny! endiamin bzw. sein Chlorhydrat.

Gibi man zu $10 \mathrm{ccm}$ dest. Wasser $0,25 \mathrm{ccm}$ einer Adrenalinlösung 1:1000, erhitzt bis zum Kochen und fügt einige Tropten einer 1 proz. Goldchloridlösung hinzu, so zeigt sich eine rote Goldkolloidreaktion. Das Adrenalin wird bekanntlich als ein Derivat der Dioxyphenyl-uMethylamino - $\beta$ - Oxypropionsaure aufgefaßit. Durch Abspaltung von Kohlensäure aus der genannten Säure soll es gebildet werden. Die Bildung im Tierkörper geschehe aber vermutlich aus 1-Tyrosin (also der $\pi$-Oxyphenyl-Aminopropionsäure), welches wieder aus der Spattung der Proteine entsteht. Das Tyrosin verwandle sich im Tierkörper zuerst in Oxyphenylaethylamin und nachher in Adrenalin. Eine Verwandschaft des Tyrosins mit dem Adrenalin wäre wohl möglich, da auch Tyrosin stahlblaue Goldkolloidreaktion auslöst. Diese Reaktion findet nicht statt, wenn Trichloressigsäure hin. zugefügt worden ist. Ich mache diese leiztere Angabe, weil sie für die Identifizierung. von Kolloidwirkungen der betreffenden und anderer Körpersubstanzen von Bedeutung sein kann. lch für meine Person vermute aber, dab das Adrenalin aus EiweiBkörpern, also Ảminosäuren, durch den LebensprozeB der Nebennierenzellen direkt hervorgebracht wird oder dab diese Zellen bereits aus dem Muskel oder den anderen endokrinen Drüsen und Organen stammende Produkte zugefihnt werden iach reduzierender Art), welche hier durch die Nebenniere zwecks weiterer Reduktionswirkung dem Blute zugeführt werden zur Ausübung der spezifiscien physiologischen reduktiven Körperfunktionen.

Eine zweite bedeutsame Kolloidreaktion lie. fert uns das Alloxan bzw der Mesoxalyl-Harnstoff; er entsteht bekanntlich aus der Harnsäure durch Oxydation. Das Alloxan gibt sowohl rote wie blaue Goldkolloidreaktionen. Eine schön kirschrote Goldreaktion erhält man auf folgendem Wege: In $160 \mathrm{ccm}$ genau- 50 grädigem Wasser lost man 0,2 Alloxan frisch auf, setzt dazu $1,5 \mathrm{ccm} 1$ proz. Goldchlorid und hocht eben schnell. Beim Erkalten bildet sich eine schöne kirschrote Goldkolloidlösung. Ich nenne im Folgenden diese Metallkolloid!ösungen io: Kürze halber statt Kolloid-Solutionen Kollu. tionen. Ich ubergebe die Eirwirking des Al- 
loxans auf andere Metalle, Silber usw., ferner in Alkohol usw. und alkalischen Lósungen usw. Erwähnen will ich nur hier noch, dae die Einwirkung der Harnsäure selbst auf Gold zu charakteristischen Reaktionen führt, welche ich als neue Harnreaktion in der Med. Klinik Nr. 28 (1919) geschildert habe.

3. ein weiterer Stoff, welcher wichtige Kolloidreaktionen auslöst, ist das Tannin ${ }^{1}$ ). Das Tannin spielt nicht nur im Pflanzenkörper eine bedeutende Rolla, sondern auch durch den Genub von Tes, Kaffee usw., vegetabilischer Kost, (Kamivoren), Wein usw. im tierischen Organismus. Die Reduktionskraft des Tannins ist eine ganz bedeutende. Ich gebe ein Beispiel zur Herstellung kirschroter Goldlösungen.

Fügt man 2u 100 ccm kochendem Wasser $0,5 \mathrm{ccm}$ einer $1 / 2-1$ proz. Tanninlösung, ferner 1,2 ccm einer 2 proz. kohlensauten Kalilosung und setzt dazu $0,85 \mathrm{ccm} 1$ proz. Goldlösung, so bildet sich eine sehr schöne kirschrote Goldkollution. Die Lösung mut frisch hergestellt werden. Merkwurdig ist, daf sich Tannin in der angegebenen Prozentuierung - mit Kaliumkarbonat versetzt - schon in einigen Tagen.in eine grine dem Chiorophyll ahnliche Färbung verwandelt. Nach Molisch braucht die Pflanze 2um Ergrünen unbedingt Eisen. Dieses Element geht aber nicht in das Chlorophyll-Molekül über, sondern scheint vielmehr eine geringwertige Organonagnesium-Verbindung zu sein. Eine Verwandtschaft zwischen dem Hämoglobin, der Muttersubstanz des Hämoporphyrin und dem Phylloporphyrin als Derivat des Chlorophylls wird vermutet, weil durch Reduktion aus beiden Hämopvrrol (Dimethylpyroi) erhaliten wird. Die Metrllbindungen beim. Blutfarbstoff an Eisen, beim Chlorophyll an Magnesium charakterisieren beide Stoffe. Wie dem auch $\mathrm{se}$, anzunehmen ist es, daß im Leben der Pflanze das Tannin eire ihm bisher noch nicht zugesprochene Wirkung zukommt und daB auch Farbstoffe der Pflanze in den Blutiten auf Kolloidreduktionen bzw. Kolloidreaktionen durch Tannin mit Spuren von Metallen: Fe, $\mathrm{Al}, \mathrm{Mg}, \mathrm{K}$, Na und anderen zufäiligen merallischen Beimengungen hervorgebracht werden könnten, wobei ich aber auf die hervorragenden Untersuchungen Wills $t$ ät ter's über Blutenfarbstoffe verweisen möchte. Die Reduktionskraft des Tannins spielt, indem wir es in Kathee, Tee usw. genteken, nicht allein in Darmkanal des tierscren Körpers eine Rolle, sondern

1) Letzerer Stoft ist aber beteits von Wo. Ostwald in stiner Buch "Dic Welt der vernachlasaigten ohrensionen" $\$ .22$ beschrieben worden. kann auch durch Aufnahme in die Blutbahn eine ganx erhebliche Bedeutung haben. Ich selbst habe Tanninmetallkollutionen $z u$ therapeutischen Effekten herangezogen.

Eine vierte äuferst bemerkenswerte Kolloidreaktion ist jene, welche durch Dimethylparaphenylendiamin und ähnliche Stoffe hervorgerufen wird. Ich gebe ein Beispiel:

Fügt man zu $10 \mathrm{ccm}$ dest. Wassers $3 \mathrm{ccm}$ einer frisch bereiteten Dimethylparaphenylendiaminlosung hinzu (1:1000), erhitzt die Mischung und gibt nun einige Tropfen 1 proz. Goldlösung hinzu, so entwickelt sich eine schöne an blutrot erinnernde Goldkollution. Genau der gleiche Farbton entsteht aber auch durch Hinzufügen von einigen Tropfen 2 proz. Kupferchloridlösung, desgl. wiederum durch Hinzufügen von einigen Tropfen 2 proz. Merkuronitratlösung, desgl. von einigen Tropten offizineller basischer essigsaurer Tonerde, ferner durch Hinzufïgen von Bleiwasset. Wăhrend diese eigentümlichen Kolloid-Farbreaktionen in heiper Lösung hervorgerufen werden, gibt Zusatz von einigen Tropfen 2,5 proz. Ferrisulfatoosung merkwürdigerweise schon in der Kalte eine blutrote Färbung. Auch andere Metalle, wie Silber, Uran, Cer (nitricum), Lanthan (nitricum), Didym (nitricum), Thor (nitricum und vor allem Platinchlorid geben Farbveränderwngen ähalich kolloiden Charakters. Die Kolloidnatur dieser Farbreaktionen wird weiterhin dadurch bewiesen, daf sie alle beim längeren Stehen bis zu einigen Tagen blaue Umwandlungen bilden. Ueberdies aber finden sich recht bemerkenswerte Hinblicke auf die eigenartige Kolloidität der Metallreaktionen mit Gold, Quecksilber, Silber, Kupfer, Platin, Blei, Aluminium.

Untersucht man aile diese Metallkollutionen spektralanalytisch, so sehen wir bauptsächlich zwei scharfe Absorptionsstreifen und einen zarten dritten im Spektrum auftreten, und zwar hei all den genannten Metallen gemeinsam in gelb und grïn bei Wellenlinie 565 bis 570 Millionstel Millimeter und 515 bis 520 Millionstel Millimeter. Die dritte zartere Absorption findet im Violetten statt: Die für die Spektralanalyse eventuell nötige Verdünnung der Reaktionen mu beim Eisen rasch hergestellt werden, denn bei $\mathrm{Zu}$ satz von zuviel dest. Wasser-verschwindet die Rotfärbung und die nunmehr gelbliche Lösung zeigt keinen Absorptionsstreifen. Die blauen Lösungen geben ebenfalls keine deutlichen $\mathrm{Ab}$ sorptionsspektren im sichtbaren Teil. Braune Molekularkomplexe verdecken den blauvioletten Teil des Spektrums. 
Vergleicht man das Absorptiansspektrum des Oxyhamoglobins mit dem gefundenen $A b$ sorptionsspektrum des Eisens, so sieht man, $\mathrm{da} B$ beim ersteren die Absorptionsstreifen bei 576 bis 580 und 535 bis 541 liegen. Die auffallende Aehnlichkeit der Absorptionsstreifen des Blutfarbstoffs mit denen des kolloiden Eisens lassen den Schluß erlaubt scheinen, daA es sich bel den Absorptionsstreifen des Blutfarbstoffes um die spektralanalytischen Streifen des Eisens handelt, welche nur durch die bestehende kolloide Eiweißverbindung auseinandergezerrt und verschoben sind. Dafür spricht auch das gleiche Verhalten der braunen alkalischen Hämatinlösungen, welche sich spektroskopisch ebenso verhalten wie braune Metallkolutionen. Will man dieses Faktum nicht in der geschilderten Weise bewerten, so müfte man an eine bisher unbekannte Metallverbindung bzw. on ein ganz anderes Metall denken wie Eisen, dem die spektroskopische Absorption zukäme. Dem kann aber nicht so sein, denn, wie gesagt, bieten eben alle die genannten Metalle insgesamt dieselben Absorptionsstreifen.

Der letztere Befund führt aber zu der Erkenntnis, dab eine Differenzierung der Metalle auf dem Wege spektroskopischer Absorption nicht möglich ist; ob diese Erkenntnis unsere Anschauung über die Bewertung der EmissionsSpektren vergaster Metalle andern kann, ist zunächst nicht wahrscheinlich. Auch für die dunkelbläuliche Beschaffenheit des venösen Blutes glaube ich das Verhalten der kolloiden Eisenreaktion in Parallele stellen zu können, insofern, als beim Hämoglobin durch die weitere Sauerstoffentziehung jener Eisenfarbton zur Gelturig kommt, wie wir ihn in den blauen Eisenreduktionen sehen.

Die gevannten Kolloidreaktionen der Metalle Gold,-Kupfer, Quecksilber, Platin, Eisen, Aluminium, Blei, Silber gehen ohne Unterschied des zur Realtion gebrachten Salzes vor sich, so dab sie, wie geschildert, an Salzen der Salzsăure, Schwefelsäure, Salpetersäure usw. gleichartig sich bilden. Die Säurekomponente hat also keinen EinfluB, nur die Metallkomponente.
Geht man an die Beobachtung der roten Metallösungen im Ultramikroskop von Sledento pf u. Zsigmondy, so sieht man bei Gold, Quecksilber, Kupfer, Platin, Silber im Küvettenstrom ein Aufblitzen unendlich vieler Ultramikronen, welche beim Kupfer rötlich, beim Quecksilber blendend hell empfunden werden. Die genasuere Beobachtung der Submikronen im Dunkelfeld zeigt, dab Goldkollutionen unendich viele gelbliche Goldteilchen zeigen; Quecksilber ergibt sowohl rundliche schwärzliche Submikronen wie auch gekreuzte Kristallkonfigurationen, die zwar den Lea'schen Silbcrkriställchen äbnlich sehen, aber wohl Komplexsalzbildungen sind, und zwar wahrscheinlich Di-Hg-Diphenylamin. Eine genauere Untersuchung des Gemisches im kolloiden Quecksilber mit diesen hellblizzenden Kristallen sieht: aber noch aus. Quantitativ entspricht die Menge der gefällien $\mathrm{Hg}$-Komplexe nicht dem a aferst geringen $Z_{u-}$ sat $z$ von $3 \mathrm{mg}$ Diphenylparaphenylendiamin zu zehn Tropfen 2 proz. Merkuronitratlôsung.

Weitere Phenylenderivate bin ich dabei zu untersuchen.

Aus den geschilderten Kolloidreduktionen sehen wir, dat gewisse Diamine eipe besonders starke Reduktionskraft haben usd wabrscheinlich mit den Reduktionskörpen: der Aininogruppen im Tierorganismus auf gleiche Stufe za stellen sind, aber auch Oxygruppen zelgen wesentliche Reduktionswirkungen. Eine Systematssierung aller dieser Körper, féner eine go nawere Durchforschung der rot oder sctiwarz gefăliten Metalldispersionen wird uns neue chemische Anschauungen über Molekularkomplexe geben als auch eine Bereicherung des physikalischen Verhaltens bzw. der Farben herbeiführen.

Einen Teil der praktisch verwertbaren Reduktionen zur Herstellung kolloider Metalle habe ich unter Fatentschutz gestełlt. Das kolloide Eisen und andere Kolloidmetalle verwerte ich therapeutisch $\mathrm{zu}$ internen, subkutanen und intravenösen Injektionen. Offetbar beruht die Wirkung vieler Anăsthetika auf ihrer Reduktionswirkung im Körper. 